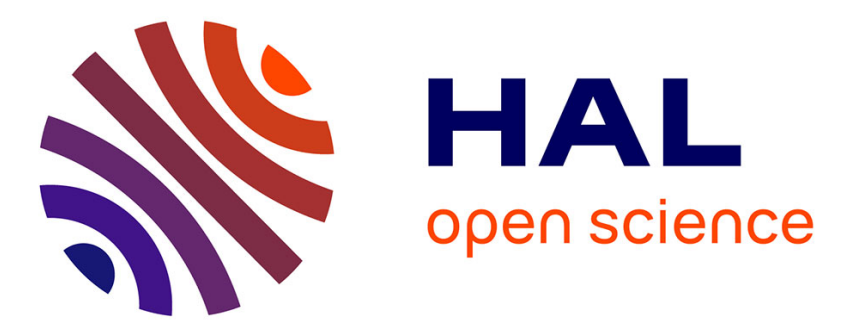

\title{
Rôle de Rhizoctonia solani Kühn dans la manifestation de certains symptômes de verse parasitaire du maïs
}

Bernard Perraton, Philippe Lucas

\section{To cite this version:}

Bernard Perraton, Philippe Lucas. Rôle de Rhizoctonia solani Kühn dans la manifestation de certains symptômes de verse parasitaire du maïs. Agronomie, 1983, 3 (8), pp.791-796. hal-00884572

\section{HAL Id: hal-00884572 \\ https://hal.science/hal-00884572}

Submitted on 1 Jan 1983

HAL is a multi-disciplinary open access archive for the deposit and dissemination of scientific research documents, whether they are published or not. The documents may come from teaching and research institutions in France or abroad, or from public or private research centers.
L'archive ouverte pluridisciplinaire HAL, est destinée au dépôt et à la diffusion de documents scientifiques de niveau recherche, publiés ou non, émanant des établissements d'enseignement et de recherche français ou étrangers, des laboratoires publics ou privés. 


\title{
Rôle de Rhizoctonia solani Kühn dans la manifestation de certains symptômes de verse parasitaire du maïs
}

Bernard PERRATON \& Philippe LUCAS

I.N.R.A., Station de Pathologie végétale, Centre de Recherches de Rennes, F 35650 Le Rheu

RÉSUMÉ

\begin{abstract}
En Vendée, dans l'Ouest de la France, il est fréquent d'observer des symptômes de verse parasitaire précoce sur maïs, liée à d'importantes nécroses racinaires. Des Fusarium, principalement $F$. oxysporum, sont très souvent isolés mais en utilisant un milieu d'isolement plus sélectif, on constate la présence très fréquente de Rhizoctonia solani.

Des essais d'inoculation au champ à différentes doses d'inoculum et à différents stades de la plante montrent le rôle de $R$. solani dans la manifestation de ce symptôme de verse.
\end{abstract}

Mots clés additionnels : Nécroses racinaires, verse précoce.

In Western France (Vendée area), maize root rot and lodging occur very frequently. Lodging appears especially on plants with severe root rot after high winds or heavy rains. The fungi which have been isolated have mostly been Fusarium oxysporum and $F$. roseum (less frequently). Rhizoctonia solani was found very exceptionally until the technique of isolation was modified.

Inoculation of maize with $F$. oxysporum neither affected plant growth nor induced root rot, but it was very easy to induce the field symptoms observed in Vendée by inoculation with $R$. solani. Various techniques have been improved and the pathogenicity of $R$. solani to maize has been shown. Isolates of this fungus belong to anastomosis group 2 (A.G. 2).

Additional key words : Root rot, early lodging.

\section{INTRODUCTION}

La verse parasitaire du maïs qui se caractérise par une pourriture des tiges et racines est généralement observée durant la période de maturation de la plante.

En France, MESSIAEN et al. (1959) considèrent que, dans la région Sud-Ouest, les espèces les plus agressives sur racines sont Pyrenochaeta terrestris (Hans) Gorenz, Phialophora radicicola Cain, Rhizoctonia solani Kühn, Pythium debaryanum Hesse, $P$. graminicola Subr. (sans possibilité d'envahir la tige), Gibberella zeae (Schw.) Petch. et Phaeocytosporella zeae Stout (avec possibilité d'envahir la tige).

Plus récemment, RouHANi et al. (1979) dressent un inventaire des champignons présents sur racines de maïs en France. Les Pythium sont abondants ainsi que Pyrenochaeta terrestris dans les régions méridionales; par contre, dans les zones septentrionales c'est Fusarium roseum (Link) Snyd. \& Hans., qui domine. En inoculation artificielle, $R$. solan $i$ se révèle le plus agressif mais il est considéré comme peu important car trop rarement isolé.
Ces auteurs limitent les causes de la verse parasitaire à deux maladies : l'anthracnose, inféodée au Sud-Ouest de la France, provoquée par Colletotrichum graminicola (Ces.) Wilson, qui attaque les tiges, et la "fusariose", observée dans toutes les zones maïsicoles de France, ainsi appelée car on isole en général des Fusarium roseum des tiges pourries. $F$. roseum (ou $F$. moniliforme Sheld dans les zones les plus chaudes) serait un parasite s'installant à la suite d'une agression parasitaire; parmi les parasites susceptibles «d'ouvrir la porte» aux Fusarium, sont cités Pythium graminicola ou $P$. arrhenomanes Drechs. et Pyrenochaeta terrestris.

Des stress ou chocs physiologiques peuvent également sensibiliser la plante à l'attaque fusarienne : ainsi quelques nuits à températures inférieures à $7{ }^{\circ} \mathrm{C}$ peuvent suffire dans le Nord de la France (BARRIERE, 1979). MESSIAEN \& BEYRIES (1981) montrent, quant à eux, que, dans le SudOuest, chaleur et sécheresse peuvent jouer le même rôle. En fait, la traduction au niveau de la plante de ces stress est une accélération de la sénescence s'accompagnant d'une sensibilité accrue des tiges à l'invasion par les Fusarium. 
En Vendée, dans l'Ouest de la France, depuis le début des années 1970, un autre type de verse du maïs, qui diffère des précédents par sa précocité d'apparition, prend de l'extension et inquiète de plus en plus les agriculteurs.

Cette maladie qui, en 1977 , a touché 40 p. 100 des parcelles de maiis dans le secteur Challans-Saint GillesPalluau, soit environ 2000 ha, se manifeste précocement, souvent bien avant le stade floraison.

Les premières attaques apparaissent par foyer où les plantes sont couchées sur le sol, à la suite d'intempéries. Un arrachage des plantes atteintes révèle des nécroses, principalement sur les racines de l'horizon superficiel. Ces racines sont cassantes et très ramifiées au-dessus des nécroses. Tout ou partie du système racinaire peut être détruit. L'attaque peut gagner les racines coronaires dont les extrémités se nécrosent et se recroquevillent, prenant l'aspect de crosses. A ce stade, on n'observe pas de pourritures des tiges.

En 1982, la verse s'est manifestée début juin alors que le mais était au stade 8-9 feuilles et, fin juillet, les racines coronaires étaient atteintes.

De nombreux isolements effectués, ces dernières années, à partir de racines et tiges ont révélé la présence de Fusarium oxysporum Schlecht., le plus souvent isolé, et de $F$. roseum dans une moindre proportion sans qu'il soit possible de reproduire les symptômes avec ces champignons. En 1981, à la demande du Groupement de Développement agricole de Challans, de nouvelles investigations ont été menées et less premiers isolements ont révélé de façon épisodique la présence de Rhizoctonia solani.

Cette note est le résultat d'un travail préliminaire sur la fréquence de $R$. solani dans la rhizosphère des plants de mais atteints et sur son rôle éventuel dans la manifestation de la verse observée dans cette région.

\section{MATÉRIEL ET MÉTHODE}

\section{A. Isolements}

Les isolements sont faits à partir de fragments de racines nécrosées, lavées abondamment à l'eau du robinet, désinfectées par immersion pendant $2 \mathrm{mn}$ dans une solution d'hypochlorite de sodium à $1 \mathrm{p}$. 100 ( $50^{\circ}$ chlorométriques). Après 3 rinçages à l'eau stérile, les fragments sont déposés sur milieu gélosé contenant $20 \mathrm{~g}$ d'extrait de malt, $125 \mathrm{mg}$ de sulfate de streptomycine et 50 unités de pénicilline $G$ sel de $\mathrm{K}$ par litre de milieu. L'intérêt de l'adjonction supplémentaire de bénomyl aux concentrations de 1 et 2 ppm sera discuté plus loin.

\section{B. Inoculation au champ}

L'inoculum est produit sur grairs d'orge humidifiés et stérilisés par 2 passages à l'autoclave $\left(1 \mathrm{~h}\right.$ à $\left.120^{\circ} \mathrm{C}\right)$, à $24 \mathrm{~h}$ d'intervalle. Après ensemencement du milieu et 3 semaines d'incubation à $23^{\circ} \mathrm{C}$, l'inoculum est séché à l'air libre.

L'inoculation des plantes est réalisée par dépôt des grains d'orge ainsi colonisés, soit dans la raie de semis, soit dans une raie parallèle à la raie de semis. Différentes doses d'inoculum au mètre linéaire sont utilisées.

\section{Inoculation au moment du semis}

Le dispositif expérimental adopté est un dispositif en blocs de Fisher à 4 répétitions. Chaque parcelle élémentaire est constituée de 4 rangs de $2,50 \mathrm{~m}$ de long, distants de $75 \mathrm{~cm}$ et recevant chacun 25 graines. Seules les plantes des 2 rangs centraux sont inoculées. a) Rôles respectifs de $\mathrm{R}$. solani et de $\mathrm{F}$. oxysporum dans les attaques sur maïs (variété "LG 11 »)

Le rôle de ces champignons a été apprécié par des contaminations au champ avec les inoculums suivants :

$-R$. solani isolé de maïs (souche $n^{\circ} 8185$ de la collection).

- F. oxysporum isolé de maïs.

- Mélange $R$. solani $+F$. oxysporum.

L'inoculum est déposé dans la raie de semis à la dose de $8 \mathrm{~g}$ par ligne de semis (soit environ 250 grains d'orge colonisés). Le mélange des 2 champignons est effectué en quantités égales, soit $4 \mathrm{~g}$ de chaque culture par ligne. La parcelle servant de témoin reçoit, par ligne, $8 \mathrm{~g}$ de grains d'orge stérilisés et non colonisés.

Les notations effectuées portent sur le nombre de plantes levées et vivantes, 12 et $26 \mathrm{j}$ après le semis, puis à la récolte. Les rendements sont calculés après récolte des 2 rangs centraux et les poids de 1000 grains estimés sur 4 échantillons de 100 grains.

b) Nuisibilité de R. solani sur maïs (variété " $L G 11$ »)

Pour cet essai, les contaminations sont effectuées en déposant un grain d'orge colonisé par $R$. solani,

- tous les $2 \mathrm{~cm}$, dans la raie de semis (inoculation 1),

- tous les $10 \mathrm{~cm}$, dans la raie de semis (inoculation 2),

- tous les $2 \mathrm{~cm}$, dans un sillon parallèle à la raie de semis et distant de celle-ci de $15 \mathrm{~cm}$ (inoculation 3 ).

Une $4^{c}$ parcelle ne reçoit pas d'inoculum.

Quatre mois après le semis sont notés, en fonction de chaque type d'inoculation, le pourcentage de plantes vivantes, leur taille, l'importance de la floraison femelle (p. 100 de plantes vivantes ayant formé un épi) et de la verse (p. 100 de plantes vivantes ayant versé). A la récolte, le rendement et les poids de 1000 grains sont calculés.

\section{Inoculation en végétation}

L'inoculum est déposé dans un sillon parallèle à la ligne de semis et distant de $15 \mathrm{~cm}$, lorsque les plantes ont atteint respectivement les stades 3-4 feuilles et 8-9 feuilles; dans les 2 cas, la dose utilisée est de 100 grains d'orge par mètre linéaire. Chaque inoculation est effectuée sur 2 rangs de 100 plantes chacun, situés dans une parcelle de grande culture (variété « Sanora»).

Des notations effectuées régulièrement au cours de la végétation permettent d'apprécier l'époque d'apparition et l'intensité de la verse en fonction du stade d'inoculation. A la récolte sont notés les pourcentages de plantes, versées ou non, ayant donné un épi ainsi que les rendements moyens par plante et les poids de 1000 grains.

\section{RÉSULTATS}

\section{A. Importance de la composition du milieu d'isolement pour la mise en évidence de $R$. solani}

Quatre échantillons de racines de maïs atteints de verse sont prélevés dans une même parcelle. Des isolements sont réalisés avec ou sans désinfection des racines sur un milieu contenant ou non du bénomyl. Après incubation à $22^{\circ} \mathrm{C}$, les colonies de champignons sont identifiées et comptées. Les résultats apparaissent dans le tableau 1.

L'adjonction de bénomyl à la concentration de $1 \mathrm{ppm}$ révèle la présence de $R$. solani, obtenu très exceptionnellement dans les isolements sur milieu sans fongicide. Parmi les Fusarium, c'est $F$. oxysporum qui prédomine comme cela a 


\section{TABLEAU I}

Comparaison de quatre méthodes d'isolement à partir d'échantillons de maïs provenant d'une parcelle de la région vendéenne.

Comparison of four methods of isolation from maize samples in a field in the Vendee area.

\begin{tabular}{|c|c|c|c|c|c|}
\hline \multicolumn{2}{|c|}{ Echantillons } & \multirow{2}{*}{$\frac{\text { A }}{\text { non }}$} & \multirow{2}{*}{$\frac{B}{\text { oui }}$} & \multirow{2}{*}{$\frac{\mathrm{C}}{\text { oui }}$} & \multirow{2}{*}{$\frac{\mathrm{D}}{\text { oui }}$} \\
\hline & Désinfection des racines & & & & \\
\hline & $\begin{array}{l}\text { Concentration } \\
\text { en bénomyl } \\
\text { du milieu }\end{array}$ & 0 & 0 & $1 \mathrm{ppm} 2$ & $\mathrm{ppm}$ \\
\hline \multirow{3}{*}{ 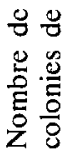 } & F. oxysporum & 19 & 20 & 27 & 1 \\
\hline & F. roseum & 10 & 8 & 3 & 1 \\
\hline & R. solani & 0 & 0 & 10 & 11 \\
\hline
\end{tabular}

été le cas dans les isolements effectués avant 1981 et contrairement à ce qui a été observé dans d'autres régions de France (MESSIAEN et al., 1959 ; BARRIERE, 1979 ; ROUHANI et al., 1979).

Les tests d'anastomose avec les souches de référence de $R$. solani (PARMETER et al., 1969), effectués sur 2 isolats obtenus de parcelles différentes, nous ont permis de rattacher ceux-ci au groupe d'anastomose AG 2.

\section{B. Rôles respectifs de $R$. solani et de $F$. oxysporum dans les attaques sur maïs}

L'inoculation par $F$. oxysporum ne provoque ni fonte de semis, ni perte de rendement (tabl. 2). Par contre, $R$. solani provoque une fonte de semis très importante. Celle-ci est légèrement moindre lorsque le champignon est associé à $F$. oxysporum, ce qui peut simplement être un effet "dose d'inoculum ».

La verse est apparue entre les $40^{\mathrm{e}}$ et $60^{\mathrm{e}} \mathrm{j}$ après semis pour l'ensemble des plantes inoculées avec $R$. solani seul ou en mélange (fig. 1). Par la suite, ces plantes se redressent à partir du $2^{\mathrm{e}}$ entre-nœud, mais restent chétives avec absence fréquente de floraison femelle.

$R$. solani est très facilement isolé à partir des racines de plantes inoculées qui portent souvent des sclérotes (fig. 2).

\section{Nuisibilité de $R$. solani sur maïs}

\section{Inoculation au semis}

Les différents modes d'inoculation utilisés provoquent des dégâts d'intensité décroissante de l'inoculation 1 à l'inoculation 3 (tabl. 3).

L'inoculation 1, caractérisée par une forte densité d'inoculum près de la semence, provoque une mortalité élevée des plantules. Les plants qui survivent donnent un faible rendement accentué par un mauvais remplissage des grains $(P M G=179 \mathrm{~g}$ )

Les inoculations 2 et 3 ont le même effet sur la phase de remplissage des grains (PMG identiques) ; les différences de rendement observées sont dues à la plus forte pression exercée en début de végétation par l'inoculum de la méthode $n^{\circ} 2$, qui, bien qu'en quantité inférieure, est plus proche de la semence.

Notons toutefois que, si dans notre expérimentation, c'est la méthode d'inoculation $\mathrm{n}^{\circ} 3$ qui affecte le moins le rendement, c'est elle qui provoque le plus de verse et aurait rendu difficile une récolte mécanique.

\section{Inoculation en végétation}

Les attaques en cours de végétation sont également préjudiciables au rendement (tabl. 4) et peuvent provoquer d'importants symptômes de verse (fig. $1 b$ ).

La verse observée le 15 juillet sur le maïs inoculé au stade 3-4 feuilles est aussi la conséquence de très fortes pluies enregistrées les 13 et 14 juillet (respectivement 18,9 et $27,6 \mathrm{~mm}$ ). A cette date, le maïs est au stade début floraison et la taille des plantes de $1,80 \mathrm{~m}$ en moyenne.

A la notation du 23 août, 76 p. 100 de ces mêmes plantes sont redressées à partir du $2^{\mathrm{e}}$ entre-nœud, mais restent très sensibles à une poussée de la main et s'arrachent facilement, découvrant un système racinaire nécrosé. $\mathrm{A}$ la récolte, il ne reste que 58 p. 100 de plantes redressées à partir du $2^{\mathrm{e}}$ entre-nœud et 32 p. 100 sont à terre (la moitié de ces dernières ne présente pas d'épis) ; 10 p. 100 ont pourri au sol.

La verse est moins importante lorsque l'inoculation est réalisée plus tardivement (stade 8-9 feuilles).

On peut constater que les 2 types d'inoculation ont le même effet sur la phase de remplissage des grains (PMG identiques). La différence de rendement observée entre les plantes inoculées précocement et celles inoculées plus tardivement (soit 26 p. 100) correspond très exactement aux plantes versées n'ayant pas formé d'épis.

\section{TABLEAU 2}

Pourcentage de plantes vivantes, rendements, poids de 1000 grains en fonction des inoculations (moyennes de 4 répétitions) - (var. "LG $11 »)$. Percent of living plants, yield, and 1.000-seed weight after different inoculations (means of 4 replicates) - (Cv. "LG 11 ").

\begin{tabular}{|c|c|c|c|c|c|}
\hline \multirow{2}{*}{$\begin{array}{c}\text { Inoculation } \\
\text { par }\end{array}$} & \multicolumn{3}{|c|}{ Pourcentage de plantes vivantes } & \multirow{2}{*}{$\begin{array}{l}\text { Rendement } \\
\text { moyen } \\
\text { parcellaire } \\
\text { (g) }\end{array}$} & \multirow{2}{*}{$\begin{array}{l}\text { Poids de } \\
1000 \text { grains } \\
\text { (g) }\end{array}$} \\
\hline & $\begin{array}{c}12 \mathrm{j} \\
\text { après semis }\end{array}$ & $\begin{array}{c}26 \mathrm{j} \\
\text { après semis }\end{array}$ & à la récolte & & \\
\hline R. solani & 16 & 7 & 4 & 22 (a) & $184(\mathrm{c})$ \\
\hline$R$. solani $+F$. oxysporum & 36 & 19 & 10 & 20 (a) & $185(\mathrm{c})$ \\
\hline F. oxysporum & 85 & 91 & 91 & 5738 (b) & 357 (d) \\
\hline Témoin & 86 & 95 & 95 & 5824 (b) & 358 (d) \\
\hline
\end{tabular}

Les valeurs suivies de lettres différentes sont significativement différentes au seuil 0,01 .

Values followed by different letters are significantly different $(P=0.01)$. 

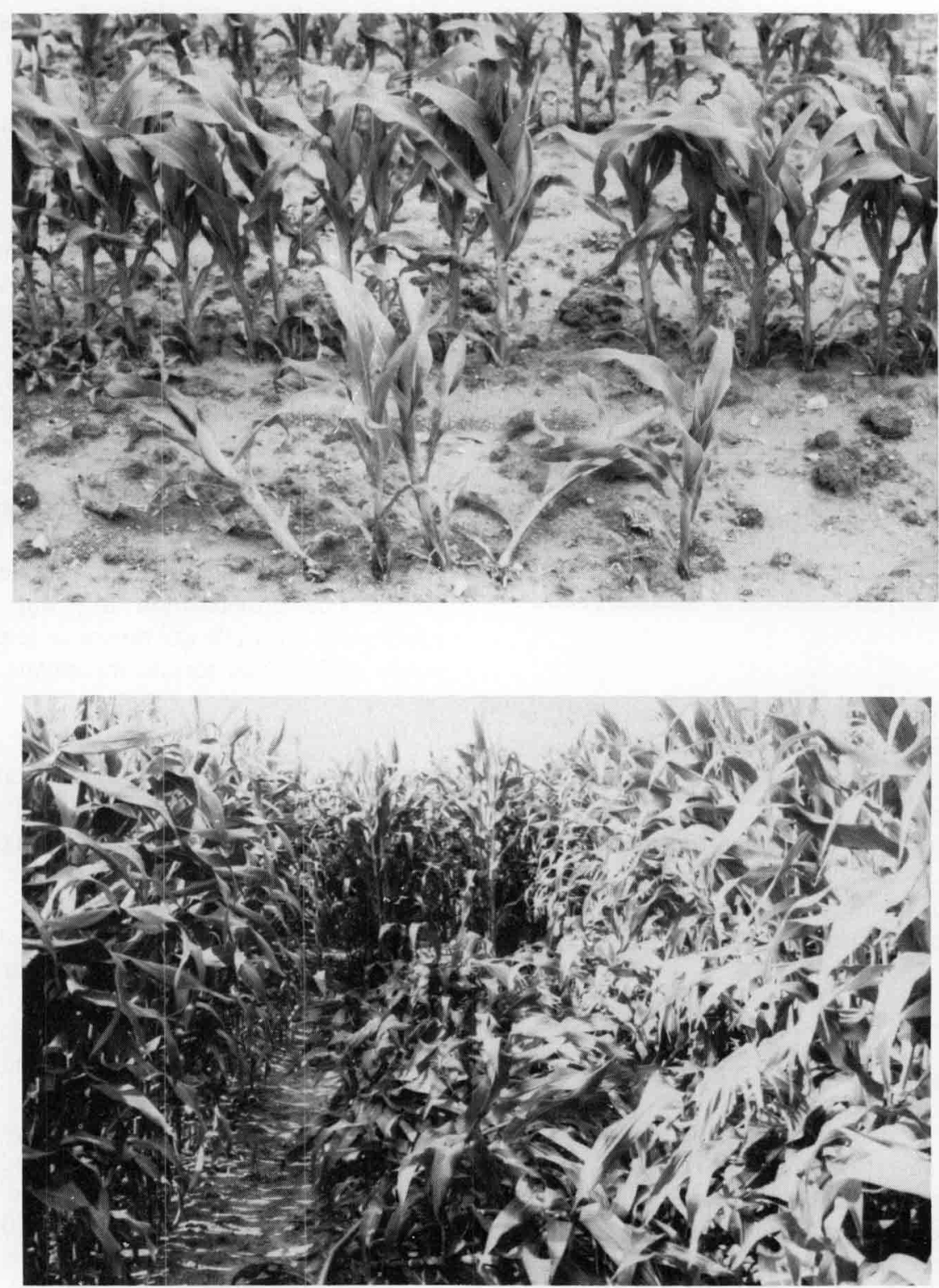

Figure 1

Verse observée au champ après inoculation avec Rhizoctonia solani. a) Inoculation au moment du semis, verse précoce (seules les plantes du rang au $1^{\text {er }}$ plan ont été inoculées).

b) Inoculation au stade 3-4 feuilles, verse observée 75 japrès semis (54 japrès inoculation) à la suite de fortes pluies (seules les plantes des 2 rangs du milieu ont été inoculées; au fond, parcelle témoin sans inoculation).

\section{DISCUSSION, CONCLUSION}

L'adjonction de bénomyl dans le milieu d'isolement permet donc de mettre en évidence la présence de $R$. solani dans la rhizosphère des plants de mais atteints de verse dans la région vendéenne. Il apparaît, d'autre part, que si $F$. oxysporum est le plus fréquemment isolé, il ne semble avoir aucune responsabilité dans les symptômes observés au champ, essentiellement dus à $R$. solani. Cette agressivité de $R$. solani avait été montrée par RoUHANi et al. (1979) et son rôle peut être minimisé du fait de la faible fréquence d'isolement. Celle-ci était-elle due à sa faible représentativité ou à une technique d'isolement inappropriée? A
Lodging in the field after inoculation with $\mathrm{R}$. solani. a) Inoculation at sowing time, early lodging (only plants in the foreground have been inoculated).

b) Inoculation at 3-4-leaf stage; lodging was assessed 75 days after sowing (54 days after inoculation) and after heavy rainfall.

l'inverse, ces mêmes auteurs avaient très fréquemment isolé Pythium spp. capables de provoquer des nécroses sur le système racinaire. Or, notre méthode d'isolement n'est pas susceptible de mettre en évidence les Pythium. Leur participation éventuelle à la nécrose des racines en Vendée ne peut donc êtrc exclue, d'autant moins que des isolements réalisés à d'autres occasions, à partir d'échantillons provenant de la région, ont révélé la présence de Pythium arrhenomanes (MESSIAEN, comm. pers., 1983).

Lès inoculations au champ dès le semis, entraînent la de jeunes plantules, si la dose d'inoculum est importante. Il ne semble pas que de tels dégâts aient été observés au champ en conditions naturelles. 

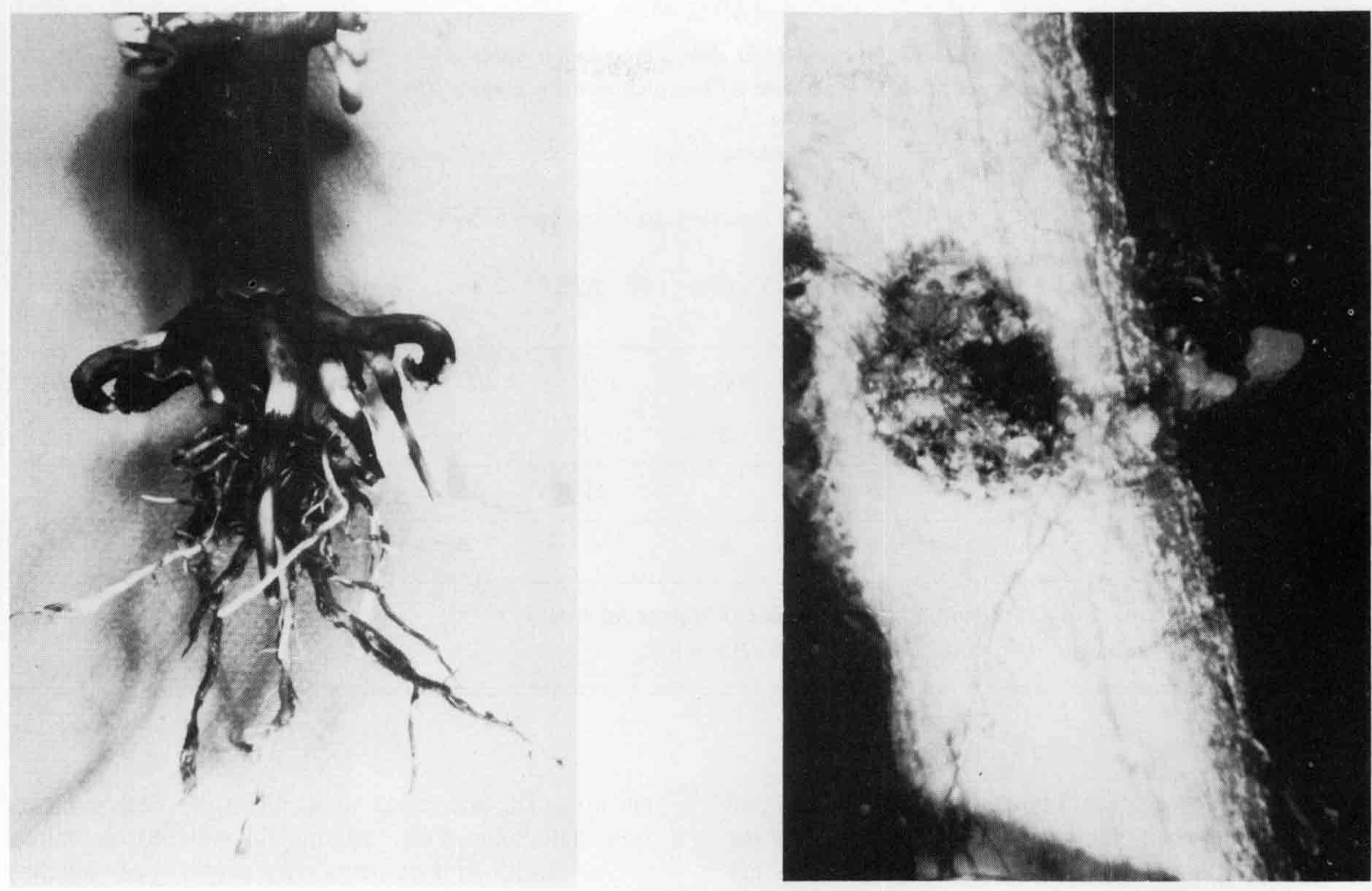

Figure 2

Nécroses du système racinaire d'un pied de maïs attaqué par Rhizoctonia solani.

a) Attaque grave, système racinaire entièrement nécrosé, racines d'ancrage en forme de crosses.

b) "Sclérote " et filaments mycéliens de R. solani à la surface d'une racine de maïs.
Root rot on maize plant attacked by $\mathrm{R}$. solani.

a) Severe crown and brace root rot - brace roots are crook shaped. b) Sclerotia and hyphae of $\mathrm{R}$. solani on a maize root.

TABLEAU 3

Pourcentage de plantes vivantes, taille, floraison, verse et rendements observés après inoculation avec $\mathbf{R}$. solani (moyennes de 4 répétitions) (Var. « LG $11 »)$.

Percent of living plants, height, flowering, lodging and yields observed after inoculation with $\mathrm{R}$. solani (means of four replicates) $-(C v$. " $L G 11 ")$.

\begin{tabular}{|c|c|c|c|c|c|c|c|c|}
\hline \multirow[b]{4}{*}{ Inoculation } & \multicolumn{6}{|c|}{ Observations en végétation 4 mois après semis } & \multicolumn{2}{|c|}{ Récolte } \\
\hline & \multirow{3}{*}{$\begin{array}{l}\text { p. } 100 \text { de } \\
\text { plantes } \\
\text { vivantes }\end{array}$} & \multicolumn{5}{|c|}{ Pourcentage de plantes vivantes } & \multirow{3}{*}{$\begin{array}{l}\text { Rendements } \\
\text { par parcelle } \\
\text { (g) }\end{array}$} & \multirow{3}{*}{$\begin{array}{l}\text { Poids de } \\
1000 \text { grains } \\
\text { (g) }\end{array}$} \\
\hline & & \multicolumn{3}{|c|}{ dont la taille est } & \multirow{2}{*}{$\begin{array}{l}\text { présentant } \\
\text { un épi }\end{array}$} & \multirow{2}{*}{$\begin{array}{l}\text { atteintes } \\
\text { par } \\
\text { la verse }\end{array}$} & & \\
\hline & & $<1 \mathrm{~m}$ & 1 à $2 \mathrm{~m}$ & $>2 \mathrm{~m}$ & & & & \\
\hline $\begin{array}{l}\text { Sur le rang } \\
\text { tous les } 2 \mathrm{~cm}\end{array}$ & 16 & 32 & 65 & 3 & 63 & 41 & 374 (a) & 179 (a) \\
\hline $\begin{array}{l}\text { Sur le rang } \\
\text { tous les } 10 \mathrm{~cm}\end{array}$ & 61 & 18 & 81 & 1 & 77 & 58 & $1500(\mathrm{~b})$ & 198 (b) \\
\hline $\begin{array}{l}\text { Sur rang à } 15 \mathrm{~cm} \\
\text { tous les } 2 \mathrm{~cm}\end{array}$ & 87 & 3 & 91 & 6 & 79 & 79 & 2450 (c) & 199 (b) \\
\hline Non inoculé & 97 & 0 & 63 & 37 & 97 & 0 & $3874(d)$ & $218(\mathrm{c})$ \\
\hline
\end{tabular}

Les chiffres suivis de lettres différentes sont significativement différents au seuil 0,01.

Values followed by different letters are significantly different $(P=0.01)$. 
TABLEAU 4

Evolution de la maladie en fonction de la date d'inoculation après semis (Var. "SANORA "). Disease progress in relation to date of inoculation after sowing ( $C v$. "SANORA").

\begin{tabular}{|c|c|c|c|c|c|c|c|c|c|}
\hline \multirow{2}{*}{$\begin{array}{c}\text { Date } \\
\text { de } \\
\text { semis }\end{array}$} & \multirow{2}{*}{$\begin{array}{c}\text { Date } \\
\text { d'inoculation }\end{array}$} & \multirow{2}{*}{$\begin{array}{l}\text { Stade du mais } \\
\text { à l'inoculation }\end{array}$} & \multicolumn{5}{|c|}{ Pourcentage de plantes versées } & \multirow{2}{*}{$\begin{array}{c}\text { Rendement } \\
\text { moyen } \\
\text { par } \\
\text { plante } \\
(\mathrm{g})\end{array}$} & \multirow{2}{*}{$\begin{array}{l}\text { Poids de } \\
1000 \text { grains } \\
\text { (g) }\end{array}$} \\
\hline & & & 9 juillet & 15 juillet & 30 juillet & 23 août & à la récolte & & \\
\hline \multirow{4}{*}{30 avril } & 21 mai & 3-4 feuilles & 4 & 100 & 100 & 100 & 100 & 76 (a) & 308 (a) \\
\hline & Pas d'inoc. & - & 0 & 0 & 0 & 0 & 0 & $128(b)$ & 358 (b) \\
\hline & 17 juin & 8-9 feuilles & 0 & 3 & 11 & 11 & 26 & $106(\mathrm{c})$ & $300(a)$ \\
\hline & Pas d'inoc. & - & 0 & 0 & 0 & 0 & 0 & $122(b)$ & $350(\mathrm{~b})$ \\
\hline
\end{tabular}

Les chiffres suivis de lettres différentes sont significativement différents au seuil 0,01.

Values followed by different letters are significantly different $(P=0.01)$.

Les inoculations en végétation montrent que l'attaque par $R$. solani peut intervenir sur au moins 3 composantes du rendement : le peuplement à la récolte, l'épiaison et le poids de 1000 grains. Dans les conditions de l'essai, une attaque précoce provoque 100 p. 100 de verse, réduit le peuplement à la récolte, l'épiaison et le poids de 1000 grains; une attaque plus tardive (inoculation au stade 8-9 feuilles) ne provoque que $26 \mathrm{p} .100$ de verse, mais affecte le poids de 1000 grains dans les mêmes proportions que l'attaque plus précoce.

En conditions naturelles, dans la région vendéenne, l'importance des dégâts est toujours estimée en fonction de la verse qui a sévi. Cette importance varie suivant les situations mais aussi dans le temps. Certaines années, la verse n'est pas observée ce qui ne semble pas être, au vu des résultats précédents, un gage de bon état sanitaire des cultures. En fait, cette verse n'est que l'étape ultime mais non inéluctable d'une attaque du système racinaire.

Des études sont en cours pour savoir si ces souches de $R$. solani sont spécifiques du maïs et si des souches de $R$. solani isolées d'autres plantes sont susceptibles de provoquer de tels symptômes. La souche utilisée pour le présent travail ainsi qu'une souche isolée dans une autre parcelle appartiennent au groupe d'anastomose AG 2.

Il est intéressant de noter que des dégâts similaires, mettant en cause des souches de $R$. solani appartenant également à ce groupe d'anastomose, ont été récemment observés aux USA (SUMNER \& BELL, 1982).

Reçu le 13 janvier 1983. Accepté le 18 avril 1983.

\section{RÉFÉRENCES BIBLIOGRAPHIQUES}

Barrière Y., 1979. Sélection du maïs pour la résistance à la pourriture de tiges. Etude de génotypes précoces. Ann. Amélior. Plantes, 29 (3), 289.304.

Messiaen C.-M., Beyries A., 1981. Etude des facteurs favorisant la pourriture des tiges chez le maïs en conditions chaudes et sèches. Agronomie, 1 (5), $\angle 09-411$.

Messiaen C.-M., Lafon R., Molot P., 1959. Nécroses de racines, pourritures de tiges et verse parasitaire du maïs. Ann. Epiphyt., 4, 441-474.
Parmeter J. R., Sherwood R. T., Platt W. D., 1969. Anastomosis grouping among isolates of Thanatephorus cucumeris. Phytopathology, 59, 1270-1278.

Rouhani H., Davet P, Poinso B., Beyries A., Messiaen C.-M., 1979. Inventaire et évaluation du pouvoir pathogène des composants de la microflore fongique sur racines de maïs en France. Ann. Phytopathol., 11 (1), 69-93.

Sumner D. R., Bell D. K., 1982. Root diseases induced in corn by Rhizoctonia solani and Rhizoctonia zeae. Phytopathology 72, 86-91. 